\title{
Surgical Complications in 2000 Renal Transplants
}

\author{
P. Eufrásio, B. Parada, P. Moreira, P. Nunes, S. Bollini, A. Figueiredo, and A. Mota
}

\begin{abstract}
Introduction. Renal transplantation is the best treatment for end-stage renal disease. In the last years, we have seen improvements in immunosuppressive treatment, which have allowed patients to experience a better quality of life and graft survival. Nevertheless, surgical complications remain important problems that increase morbidity, mortality, costs, and hospitalization. Our purpose was to evaluate surgical complications among a large series of 2000 renal transplantations.

Patients and Methods. We retrospectively analyzed all surgical complications among 2000 renal transplants performed between June 1980 and March 2010 in our department.

Results. Among $318(15.9 \%)$ surgical complications, $4.8 \%$ of patients had urologic problems. Ureteral stenosis and fistula, stent obstruction, and ureteral necrosis occurred in $2.7 \%, 1.8 \%, 0.1 \%$, and $0.2 \%$ of patients, respectively. Vascular complications reported in $2.7 \%$ of patients included arterial or venous thrombosis $(1.0 \%$ or $0.4 \%)$, both arterial and venous thrombosis $(0.1 \%)$, renal infarction $(0.1 \%)$, renal artery aneurysm $(0.1 \%)$ as well as arterial stenosis $(0.5 \%)$, kinking $(0.4 \%)$, or dissection $(0.1 \%)$. Other complications, not specifically related with transplantation surgery, occurred in $4.4 \%$ of patients.

Conclusion. Renal transplantation is a safe surgery by experienced teams. Our rates of surgical complications were within those reported by other series. A meticulous surgical technique is mandatory to prevent them. Prompt diagnosis and management are required to prevent graft damage and patient morbidity.
\end{abstract}

$\mathbf{R}$ ENAL TRANSPLANTATION is the best treatment for end-stage renal disease (ESRD). Continued improvements in graft survival have led to widespread acceptance of renal transplantation as the preferred treatment for the majority of patients with ESRD. ${ }^{1}$ Despite evolutions in surgical techniques and immunosuppressive regimens, surgical complications remain important clinical problems that may increase morbidity, hospitalization, and costs. Some of these complications can be life threatening; their prompt recognition and treatment can save the patient and the graft. ${ }^{2,3}$ The purpose of this study was to present our single-center experience concerning surgical complications among a large series of 2000 renal transplantations.

\section{METHODS}

We retrospectively analyzed surgical complications among 2000 renal transplantations performed in our department from June 1980 to March 2010. The mean age of the recipients was $43.3 \pm$ 13.0 years. There were 1316 (65.8\%) male and 684 (34.2\%) female patients. The kidneys came from a cadaveric donor in $96.4 \%$ of cases. Immunosuppressive protocols have varied among the various periods, usually consisting of 3-drug regimens. The surgical technique used the common iliac vessels to perform end-to-side vascular anastomoses with running sutures in the majority of patients. A Politano-Leadbetter technique was performed as the ureteroneocystostomy from 1980 to 1987, and thereafter, a LichGregoir technique with temporary ureteral stenting usually for 1 month. All patients were closely and regularly followed at our department, with a mean follow-up of $81.57 \pm 70.33$ months. At the moment, $1610(80.5 \%)$ of all patients are alive; $14.6 \%$ of them have nonfunctioning grafts. The remaining 390 patients $(19.5 \%)$ have died. Globally, $65.9 \%$ of the patients (1318) have functioning kidneys and $34.1 \%$ (682 patients), nonfunctioning ones. We divided all surgical complications into vascular, urologic, lymphatic, wound related, or other types. For statistical analyses, we used SPSS version 16.0 (SPSS, Inc., Chicago, Ill).

From the Department of Urology and Renal Transplantation, Coimbra University Hospital, Coimbra, Portugal.

Address reprint requests to Pedro Eufrásio, Department of Urology and Renal Transplantation, Coimbra University Hospital, 3000 Coimbra, Portugal. E-mail: pedrocotovio26@hotmail.com

(c) 2011 by Elsevier Inc. All rights reserved. 360 Park Avenue South, New York, NY 10010-1710 


\section{RESULTS}

Surgical complications occurred in 318 patients (15.9\%). Urologic problems were most commonly reported, followed by vascular complications, wound-related problems, hematoma, lymphoceles, and other complications.

The urologic complications in 96 patients $(4.8 \%)$ included $54(2.1 \%)$ ureteral strictures; $36(1.8 \%)$ ureteral fistulae; $4(0.2 \%)$ distal ureteral necrosis and 2 cases $(0.1 \%)$ with obstruction of the ureteral stent (Table 1). The majority of urinary fistulae were diagnosed within the first weeks after surgery and mainly managed by surgical interventions, whereas ureteral obstructions occurred from the first days to several years after transplantation. Ureteroneocystostomy was performed in the majority of these patients, although a few were managed by endoscopic incision or a temporary stent.

Overall, we discovered vascular complications in 54 $(2.7 \%)$ patients. Arterial thrombosis was the most common problem, occurring in 20 patients $(1 \%)$. Other vascular complications were arterial stenosis in $10(0.5 \%)$, venous thrombosis in $8(0.4 \%)$, and arterial kinking in another 8 patients $(0.4 \%)$. We observed graft infarction, arterial dissection, venous plus arterial thrombosis, or renal artery aneurysm in 2 patients each $(0.1 \%$; Table 2$)$. All of these patients with vascular problems were managed with open surgery.

There were 30 patients $(1.5 \%)$ who developed symptomatic or obstructive lymphoceles with alterations in graft function. Treatment was mostly conservative, namely, placement of a temporary nephrostomy tube, although a few patients underwent surgical drainage and decortication.

Wound-related problems developed in 50 cases $(2.5 \%)$. Twenty patients $(1 \%)$ had wound infections and $30(1.5 \%)$, dehiscence and hernia; the latter group mostly requiring surgical correction.

Finally, other types of surgical complications occurred in 88 patients $(4.4 \%)$, including hematoma in 42 transplants $(2.1 \%)$, bleeding in $20(1.0 \%)$, abscess in $10(0.5 \%)$, graft rupture in $6(0.3 \%)$, intestinal perforation in 2 , and hemoperitoneum in another 2 recipients. All of these patients were reoperated.

Actuarial graft survival rates at $1,3,5$, and 10 years were $90.9 \%, 84.8 \%, 78.5 \%$, and $62.5 \%$, respectively. Actuarial patient survival rates at $1,3,5$, and 10 years were $94.5 \%$, $91.1 \%, 87.3 \%$, and $76.8 \%$, respectively. Overall, 50 patients $(2.5 \%)$ lost their grafts due to surgical problems.

Table 1. Prevalence of Urologic Complications

\begin{tabular}{lc}
\hline Urologic Complications & Prevalence (\%) \\
\hline Ureteral stenosis & 2.7 \\
Ureteral fistula & 1.8 \\
Necrosis of distal ureter & 0.2 \\
Stent's obstruction & 0.1 \\
Total & 4.8 \\
\hline
\end{tabular}

Table 2. Prevalence of Vascular Complications

\begin{tabular}{lc}
\hline \multicolumn{1}{c}{ Vascular Complications } & Prevalence (\%) \\
\hline Arterial thrombosis & 1 \\
Venous thrombosis & 0.4 \\
Arterial and venous thrombosis & 0.1 \\
Renal infarct & 0.1 \\
Aneurysm of renal artery & 0.1 \\
Arterial stenosis & 0.5 \\
Kinking of artery & 0.4 \\
Arterial dissection & 0.1 \\
Total & 2.7 \\
\hline
\end{tabular}

\section{DISCUSSION}

Renal transplantation has been established to be safe. Since the first operations, surgical complications have followed this procedure, although the rates are presently lower owing to improvements in immunosuppressive regimens and refinements in operative technique. As surgical experience increases in many centers, easier and safer techniques are adopted to produce fewer complications. ${ }^{3}$ Nevertheless, renal graft survival has been related not only to protocols of immunosuppression and of donor and patient selection, but also to surgical complications and their management. ${ }^{4}$ These complications rarely lead to graft loss, with the exception of vascular problems or patient death. ${ }^{5}$

Urologic complications are the most common surgical problems after renal transplantation. Presently, their incidence varies from $2.5 \%$ to $12.5 \%{ }^{6}$ being lower than the rates at the beginning of renal transplantation, when they reached values as high as $25 \% .^{7}$ Rarely, these urologic problems lead to graft loss or patient death, but are an important cause of morbidity, delayed graft function, hospitalization, and increased costs. ${ }^{8}$ Decreased blood supply to the donor ureter and faulty surgical techniques are the main causes of urologic complications, ${ }^{9}$ but other problems such as immunosuppressive drugs, rejection, and BK virus infection are also important, especially as causes of late obstruction. Although low-dose steroid protocols and meticulous operative technique tend to reduce its incidence, ${ }^{10}$ poor ureteral vascularization owing to the increasing use of expanded criteria donors, namely older individuals, have tended to increase this rate. As observed in our department, ${ }^{11}$ the technique of ureteral anastomosis does not seem to influence the incidence of complications. We have mainly adopted the extravesical Lich-Gregoir technique with an anti-reflux mechanism. The incidence of urologic problems in our series was comparable with others' experiences. Ureteral stenosis and fistula are the main causes; their management has mostly consisted of open surgical correction.

Vascular complications are less frequent, with incidence rates from $1 \%$ to $23 \% .^{12}$ Generally, these problems have a devastating course, especially arterial or venous thrombosis, with graft loss in the majority of patients. Arterial stenosis is considered to be the most common vascular complication $^{13}$; it can result from an inadequate suture technique, 
renal artery trauma, kinking of the artery, vascular-type rejection, and atherosclerosis of the donor or the recipient arteries. Its management consists of surgical revision or percutaneous transluminal angiography with balloon dilatation or placement of an endovascular stent. Arterial thrombosis is a rare complication, representing a major cause of graft loss in the early posttransplant period. It generally results from operative technique problems, although other causes can be implicated, such as hypotension and coagulation disorders; immediate surgical thrombectomy with revision of the anastomosis is the rule. Venous thrombosis is another rare complication after renal transplantation. It is generally an early postoperative event, representing one of the most common causes of early graft loss. The etiology is often multifactorial, but once again, technical problems are usually present. Surgical exploration with revision of the anastomosis is almost always required and, as in arterial thrombosis, it is necessary to perform a graft nephrectomy in some cases. Other vascular complications occurring in the postoperative period are hematomas, generally small and asymptomatic and not requiring surgery, and anastomotic aneurysmal formation, which is uncommon, with a prevalence of $<1 \%$. The last problem may require surgical excision. In our series, arterial thrombosis was the most common vascular complication, with a prevalence of $1 \%$. All patients with vascular problems were reoperated; in some cases the graft was lost, mainly as a consequence of arterial or venous thrombosis. Once again, our rates of vascular complications were within the range reported in the literature.

Lymphoceles occur mainly due to the vascular dissection that opens lymphatic vessels. In the majority of patients, the fluid collections are asymptomatic, an incidental finding on ultrasound examination that requires no treatment. When larger collections are present or associated with dilatation of the collecting system, pain, fever, or an unexplained decline in renal function, then ultrasound-guided aspiration is indicated. Sometimes, it is necessary to place a nephrostomy tube. If an uninfected lymphocele recurs, it is usually treated by unroofing into the peritoneal cavity by either open or laparoscopic surgery. In our series, the rate of symptomatic or complicated lymphoceles was $1.5 \%$; the treatment was mostly conservative. Wound-related problems, such as infection and hernia formation, are higher than in other surgeries owing to the negative effect of immunosuppressive drugs on healing. Most cases of suture infection have been managed medically. For cases of hernia in the abdominal wall, the majority of patients underwent hernioplasty. Our rates of infection and hernia were, $1.0 \%$ and $1.5 \%$, respectively.

Other complications were present in $4.4 \%$ of patients. Generally, they were not specifically associated with transplant surgery. They included cases of hematoma, mostly requiring reoperation, bleeding necessitating laparotomy, abscess, intestinal perforation, and hemoperitoneum.

In conclusion, surgical complications among our series of 2000 renal transplants were within the range of other series. It is a safe procedure in our department, based on patient and graft survivals, with a very low rate of graft loss owing to surgical problems. Early diagnosis and management can decrease the morbidity usually associated with these complications, potentially saving the graft and the patient.

\section{REFERENCES}

1. Ojo AO, Hanson JA, Meier-Kriesche H, et al: Survival in recipients of marginal cadaveric donor kidneys compared with other recipients and wait-listed transplant candidates. J Am Soc Nephrol 12:589, 2001

2. Karakayali H, Emiroglu R, Sevmis S, et al: Postoperative surgical complications in renal transplant recipients: one center's experience. Transplant Proc 33:2683, 2001

3. Loughlin KR, Tilney NL, Richie JP: Urologic complications in 718 renal transplant patients. Surgery 95:297, 1984

4. Hobart MG, Streem S, Gill I: Renal transplant complications: minimally invasive management. Urol Clin North Am 27:787, 2000

5. Hashimoto Y, Nagano S, Oshima S: Surgical complications in kidney transplantation: experience from 1200 transplants performed over 20 years at six hospitals in central Japan. Transplant Proc 28:1465, 1996

6. Emiroglu R, Karakayali H, Seumis S, et al: Urologic complications in 1275 consecutive renal transplantations. Transplant Proc 33:2016, 2001

7. Starzl TE, Groth CG, Putman CW, et al: Urological complications in 216 human recipients of renal transplants. Ann Surg $172: 1,1970$

8. Coltry AJ Jr, Schlegel JU, Lindsey ES, et al: Urological complications in renal transplantation. J Urol 112:564, 1974

9. Shoskes DA, Hanbury D, Cranston D, et al: Urological complications in 1000 renal transplant recipients. J Urol 153:18; 1995

10. Parada B, Figueiredo A, Mota A, et al: Surgical complications in 1000 renal transplants. Transplant Proc 35:1085, 2003

11. Moreira P, Parada B, Figueiredo A, et al: Comparative study between two techniques of ureteroneocystostomy: Taguchi and Lich-Gregoir. Transplant Proc 39:2480, 2007

12. Fervenza FC, Lafayette RA, Alfrey EJ, et al: Renal artery stenosis in kidney transplant. Am J Kidney Dis 31:142, 1998

13. Aguera Fernandez LG, Zudaire JJ, Isa Wa, et al: Vascular complications in 237 recipients of renal transplant from cadaver. Actas Urol Esp 16:292, 1992 